\title{
Electrical and structural characteristics of yttrium oxide films deposited by rf-magnetron sputtering on $\boldsymbol{n}$-Si
}

\author{
E. K. Evangelou, ${ }^{\text {a) }}$ C. Wiemer, and M. Fanciulli \\ Laboratorio MDM-INFM, Via C. Olivetti 2, 20041 Agrate Brianza, Milano, Italy \\ M. Sethu and W. Cranton \\ Center for Creative Technology, Nottingham Trent University, Nottingham NG1 4BU, United Kingdom
}

(Received 25 November 2002; accepted 17 April 2003)

\begin{abstract}
Yttrium oxide dielectric films were grown by rf-magnetron sputtering on $n$ - $\operatorname{Si}(100)$ substrates and annealed in vacuum at temperatures ranging from 400 to $600^{\circ} \mathrm{C}$. The main aim of this work was the investigation of the interface between the dielectric film and silicon. Both structural (x-ray diffraction and transmission electron microscopy) and electrical characterization were used for this purpose. No structural change was observed on the interfacial native oxide layer after annealing at $600^{\circ} \mathrm{C}$ for $1 \mathrm{~h}$. Metal-oxide-semiconductor structures defined by the evaporation of $\mathrm{Al}$ electrodes show low leakage currents, moderate dielectric constant (around 14), and high densities of positive charges trapped in the oxide. Hysteresis effects in capacitance-voltage $(C-V)$ curves reduce with the annealing temperature. Another interesting observation is the parallel shift of the $C-V$ curves along the voltage axis with frequency. An insulator trap model is proposed to explain this behavior. (C) 2003 American Institute of Physics. [DOI: 10.1063/1.1580644]
\end{abstract}

\section{INTRODUCTION}

The enormous growth and the significant progress of information technology in the modern world are based to a very large extent on the $\mathrm{SiO}_{2} / \mathrm{Si}$ interface properties. The industry's demand for greater integrated circuit functionality and performance at lower cost requires an increased circuit density, which is translated into a higher density of transistors on a wafer. Currently, this need is satisfied by introducing advanced materials in the gate of the transistors. By employing nitride/oxynitride gate stacks, functional complementary metal-oxide-semiconductor (CMOS) transistors with physical gate thicknesses $<4 \mathrm{~nm}^{1,2}$ have been demonstrated. Unfortunately, although Si microelectronic devices will be manufactured with $\mathrm{SiO}_{2}$ and $\mathrm{Si}-\mathrm{O}-\mathrm{N}$ for the foreseeable future, continued scaling of integrated circuit devices will necessitate the introduction of an alternate gate dielectric once the $\mathrm{SiO}_{2}$ gate dielectric thickness approaches $\sim 1.5 \mathrm{~nm}$. Indeed, the extremely small physical thickness typically required for the gate dielectric results in large leakage current, which is expected to increase power consumption. Excessive gate leakage may be avoided by using high- $k^{1-3}$ gate dielectrics (instead of $\mathrm{SiO}_{2}$ ), which can provide low equivalent oxide thickness $(\mathrm{EOT}<1 \mathrm{~nm})$ at relatively large (typically $>4 \mathrm{~nm}$ ) physical thickness.

Among the various extrinsic dielectrics $\mathrm{Ta}_{2} \mathrm{O}_{5}, \mathrm{HfO}_{2}$, $\mathrm{ZrO}_{2}, \mathrm{TiO}_{2}, \mathrm{SrTiO}_{3}, \mathrm{BaSrTiO}_{3}(\mathrm{BST})$, and $\mathrm{Y}_{2} \mathrm{O}_{3}$ have been investigated extensively. A number of detailed reviews and articles on the subject have been published recently. ${ }^{1-4}$ Despite some prerequisites having been met by these dielectrics, their device fabrication and performance potential has not yet been fully realized.

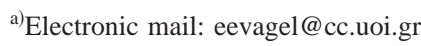

Yttrium oxide deposited on silicon by rf-magnetron sputtering, ${ }^{5-7}$ electron-beam evaporation, ${ }^{8-10}$ laser ablation and ion beam sputtering, ${ }^{11,12}$ as well as by means of epitaxy ${ }^{13,14}$ has been proposed as a candidate oxide to replace $\mathrm{SiO}_{2}$. The low lattice mismatch between the $\mathrm{Si}$ lattice parameter $\left[a_{0}(\mathrm{Si}) \times 2=1.086 \mathrm{~nm}\right]$ and that of the $\mathrm{Y}_{2} \mathrm{O}_{3}$ $(1.064 \mathrm{~nm})$, the high thermodynamic compatibility, ${ }_{15}$ and the relatively high conduction band offset $(\sim 2.3 \mathrm{eV})^{16}$ with $\mathrm{Si}$ are among the interesting properties of this material. Although amorphous or polycrystalline $\mathrm{Y}_{2} \mathrm{O}_{3}$ exhibit good electrical characteristics, epitaxial $\mathrm{Y}_{2} \mathrm{O}_{3}$ is not of adequate structural and electrical quality yet. ${ }^{17}$

A major problem for the use of these oxides is the extremely poor electrical characteristics of the interface between the extrinsic dielectrics and the silicon substrate. Another one is the high density of defects in the extrinsic dielectrics. In many cases the interface defects arise from an interfacial silicon oxide $\left(\mathrm{SiO}_{x}\right)$ layer, which grows unintentionally due to oxygen diffusion during the deposition of the oxide and also during postanneal or further postprocessing. This high transport of oxygen is also responsible for the high density of oxygen vacancies in the bulk of some oxides (e.g., $\mathrm{ZrO}_{2}$ and $\mathrm{HfO}_{2}$ ) that leads to an increased amount of positive trapped charge. To overcome these problems, attempts have been made to reduce the randomly occurring microdefect density in the bulk dielectric by improving the oxide stoichiometry using postdeposition oxygen annealing. In another approach, a bilayer dielectric structure consisting of a thin $\mathrm{SiO}_{2}$ layer at the interface between the extrinsic dielectric and $\mathrm{Si}$ was found to be extremely effective in improving the dielectric strength and reducing the leakage currents. ${ }^{3}$ The effect of this interfacial layer on the electrical properties of various alternative gate dielectrics has been studied extensively during the last few years. ${ }^{3,4,18}$ In a recent publication 
on electron-beam evaporated ultrathin $\mathrm{Y}_{2} \mathrm{O}_{3}$ films ${ }^{17}$, it has been reported that annealing in vacuum can eliminate the interfacial native oxide layer leading to good structural and electrical properties.

In the present work, we report on the electrical and structural studies of thick $(340 \mathrm{~nm}) \mathrm{Y}_{2} \mathrm{O}_{3}$ films grown by rf-magnetron sputtering and subjected to postannealing treatment at 400,500 , and $600{ }^{\circ} \mathrm{C}$ for $1 \mathrm{~h}$ in vacuum. The electrical results are correlated to the structural results from x-ray diffraction (XRD) and transmission electron microscopy (TEM) measurements and show that annealing in vacuum can indeed be used in order to avoid the formation of a thick $\mathrm{SiO}_{x}$ layer. In addition we studied the effect of uniformly distributed bulk oxide traps on the measured high frequency capacitance-voltage $(C-V)$ curves.

\section{EXPERIMENTAL DETAILS}

For this investigation, $\mathrm{Y}_{2} \mathrm{O}_{3}$ films were deposited by $\mathrm{rf}$ magnetron sputtering in argon atmosphere using two independent custom-built sputtering systems. The pregrowth chamber base pressure was nominally $2.0 \times 10^{-7} \mathrm{mbar}$ and during deposition the substrate temperature and argon pressure were maintained at approximately $190{ }^{\circ} \mathrm{C}$ and $3 \mathrm{mbar}$, respectively. The films were deposited onto $100 \mathrm{~nm}$ diameter $n$-type $\mathrm{Si}$ (100) single crystal substrate of nominal resistivity 1-5 $\Omega \mathrm{cm}$. During deposition the wafers were rotated to insure uniform film growth. Prior to deposition, the substrates were prebaked for $20 \mathrm{~min}$ at $700^{\circ} \mathrm{C}$. Deposition plasma is created by applying $120 \mathrm{~W}$ rf power to the $7.5 \mathrm{~cm}$ diameter target positioned $15 \mathrm{~cm}$ away from the substrate. The source material is a $\mathrm{Y}_{2} \mathrm{O}_{3}$ solid target with a nominal purity of 99.9\% manufactured by Cerac Inc. Postdeposition annealing at 400,500 , and $600{ }^{\circ} \mathrm{C}$ for $60 \mathrm{~min}$ was also performed in the same chamber and in vacuum under $2.0 \times 10^{-7}$ mbar using a substrate heater. In order to maintain minimum growth tolerance between different annealed samples, the $100 \mathrm{~mm}$ wafers were cleaved to halves or quarters and each piece annealed individually.

The microstructure and crystalline nature of as-deposited and annealed films were studied by transmission electron microscopy (TEM) performed in a LEO $992 \Omega$ microscope equipped with a $\Omega$-type in column energy filter operated at $200 \mathrm{kV}$. In order to reduce ion beam induced surface amorphization, the $\mathrm{Ar}^{+}$ion energy was kept as low as $3.5 \mathrm{keV}$. A $10 \mathrm{eV}$ wide and zero loss peak centered slit was used to exclude the contributions from plasmon losses $(\Delta E$ $>5 \mathrm{eV})$.

$\mathrm{X}$-ray diffraction was performed on a system equipped with a position sensitive detector able to collect data over $120^{\circ}$ at the same time. The radiation source was an $\mathrm{x}$-ray tube operating at $1.2 \mathrm{~kW}$ and equipped with a multilayer monochromator able to select a parallel beam of $\mathrm{Cu} K \alpha$ radiation. Diffractograms were collected in both grazing incidence and Bragg-Brentano $(\theta-2 \theta)$ geometry.

Metal-insulator-semiconductor (MIS) diodes were formed by the thermal evaporation of aluminum (Al) electrical contacts on the surface of the thin film. Approximately $200 \mathrm{~nm}$ thick Al was evaporated in vacuum under 1

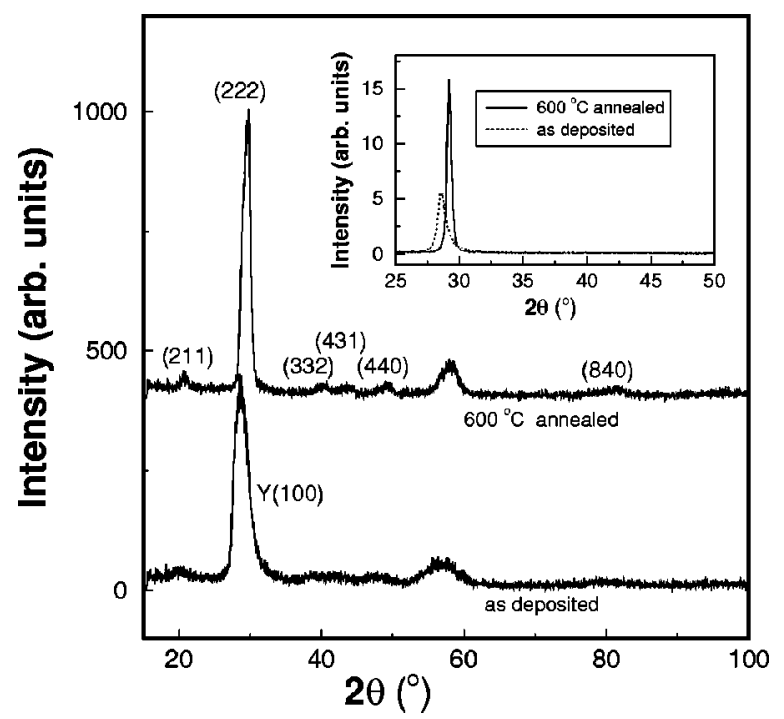

FIG. 1. Grazing incidence XRD patterns of the $\mathrm{Y}_{2} \mathrm{O}_{3}$ films. The corresponding Bragg-Bentano analysis is shown in the inset.

$\times 10^{-6}$ mbar through an appropriate mask to delineate a circular contact area of $0.785 \mathrm{~mm}^{2}$. The top contacts were deposited immediately after annealing. Ohmic back contact was also made by thermal evaporation of $300 \mathrm{~nm}$ thick Al.

The $C-V$ measurements were performed in a probe station using a HP 4284A $L C R$ meter. $I-V$ curves were also taken in the same probe station using a HP 4140B picoammeter.

\section{RESULTS AND DISCUSSION}

\section{A. X-ray diffraction (XRD)}

$\mathrm{X}$-ray diffraction data of the as-deposited and $600{ }^{\circ} \mathrm{C}$ annealed samples are presented in Fig. 1. The diffractograms taken at grazing incidence (angle of incidence $\theta=0.5^{\circ}$ ) show the presence of peaks for both the annealed and asdeposited samples. The increase of the scattered intensity at $2 \theta \sim 57^{\circ}$ is related to the (311) Bragg reflection of the (100) silicon substrate that starts to be excited at such a low angle. In the as-deposited sample, one broad peak is found around $2 \theta=30^{\circ}$ and several features are present at $20^{\circ}, 40^{\circ}$, and $47^{\circ}$ suggesting a polycrystalline nature for this sample. The peak can be indexed as belonging to the (100) reflection of hexagonal yttrium (REF: PDF33-1458). The position of the peak is shifted as compared to the bulk values suggesting a distortion of the hexagonal lattice. This distortion can be related to either oxygen inclusions in the yttrium lattice or to ordered $\mathrm{Y}_{2} \mathrm{O}_{3}$ crystallites causing the diffraction pattern to be actually related to the superposition of the reflections of yttrium and of $\mathrm{Y}_{2} \mathrm{O}_{3}$ in agreement with the presence of the other features. In the $600^{\circ} \mathrm{C}$ annealed sample, peaks are found at $20^{\circ}, 30^{\circ}, 40^{\circ}, 45^{\circ}$, and $50^{\circ}$. These peaks are sharper than the ones found in the as-deposited sample suggesting a better crystallization of the sample. The reflections are identified as belonging to the $\mathrm{Y}_{2} \mathrm{O}_{3}$ cubic phase (REF: PDF 25-1200) and correspond to a polycrystalline sample. The analysis in Bragg-Brentano geometry is presented in the inset of Fig. 1. Only one peak is found in the analyzed $2 \theta$ range for both 
as-deposited and annealed samples. This suggests a preferential orientation of the crystallites with the (100) planes of hexagonal yttrium and the (222) planes of cubic $\mathrm{Y}_{2} \mathrm{O}_{3}$ parallel to the substrate. The samples annealed at the intermediate temperatures of 400 and $500{ }^{\circ} \mathrm{C}$ were also analyzed by grazing incidence diffraction. Their diffraction patterns (not shown here) present just one peak related to the $\mathrm{Y}_{2} \mathrm{O}_{3}$ (222) reflection. The position and the full width at half maximum of this peak are between the two extreme cases presented in Fig. 1 for the as-deposited sample and the sample annealed at the higher temperature.

Fukumoto et al. ${ }^{19}$ deposited $\mathrm{Y}_{2} \mathrm{O}_{3}$ films on a $\mathrm{Si}(100)$ substrate at a temperature $T_{d}=800{ }^{\circ} \mathrm{C}$ by electron-beam evaporation from $\mathrm{Y}_{2} \mathrm{O}_{3}$ grains in oxygen atmosphere. Their XRD results indicated that $\mathrm{Y}_{2} \mathrm{O}_{3}$ has (222) preferentially oriented planes parallel to the $\mathrm{Si}(100)$ plane. However, Harada et al. ${ }^{20}$ reported that $\mathrm{Y}_{2} \mathrm{O}_{3}$ films deposited at $T_{d}=820^{\circ} \mathrm{C}$ also by electron-beam evaporation on clean $\mathrm{Si}(100)$ have preferentially oriented the (110) planes, in accordance with the results of Choi et al. ${ }^{21}$ who deposited their films by the reactive ionized cluster beam deposition technique. Both groups try to explain this difference by means of the existence of a native $\mathrm{SiO}_{2}$ layer.

In another approach Dimoulas et al. ${ }^{10,17}$ have reported the growth of thin $\mathrm{Y}_{2} \mathrm{O}_{3}$ films by electron-beam evaporation directly on Si (100) substrates in a molecular beam epitaxy system under UHV conditions. Depending on the deposition temperature they observe the appearance of both (110) and (111) preferential orientations. At low temperature the (111) orientation prevails, at medium (around $300{ }^{\circ} \mathrm{C}$ ) temperatures they coexist, while at higher temperatures only peaks corresponding to the (110) planes are present. The authors do not attempt to correlate their findings to any specific deposition conditions, as the parameters involved are many and complicated.

Finally in an earlier work by Horng et al. ${ }^{6}$ on thick (200-300 nm) $\mathrm{Y}_{2} \mathrm{O}_{3}$ films grown on $p^{+}$type (100) substrates by rf magnetron sputtering, the same diffused peak appears for the as-deposited films. After annealing though, both (111) and (100) planes appear especially after rapid thermal annealing at $850^{\circ} \mathrm{C}$.

In any case the ideal "cube on cube" growth seems to be a difficult task. This is also the situation in this work, which shows evidence of the (111) growth direction with a pronounced mosaic texture in good agreement with several recent works devoted to the $\mathrm{Y}_{2} \mathrm{O}_{3}$ thin-film deposition on $\mathrm{Si}$ substrates. One explanation is the fact that the (111) plane of the $\mathrm{Y}_{2} \mathrm{O}_{3}$ is the cleavage plane of this structure and therefore the lowest surface energy plane. The interfacial layer of amorphous $\mathrm{SiO}_{2}$ on top of the $\mathrm{Si}$ substrate is often invoked to explain this growth direction, based on a minimization of surface energy. ${ }^{22}$

\section{B. Transmission electron microscopy}

As one of the most critical issues for the usefulness of the alternative gate dielectrics is the oxide/Si interface, TEM measurements are often used to investigate the quality of this interface. Out of the four examined films, we show in Figs. 2

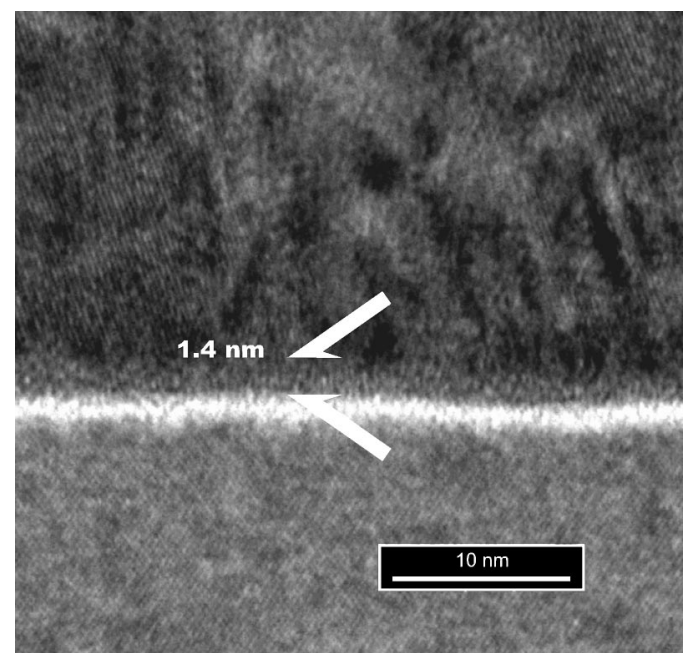

FIG. 2. HRTEM picture of the as-deposited film. The thickness of the native oxide is estimated around $1.4 \mathrm{~nm}$.

and 3 the Si (110) cross-sectional bright field images of the as-deposited and the $600{ }^{\circ} \mathrm{C}$ annealed films. The latter was chosen as it showed the best electrical characteristics (as discussed later in Sec. IIIC). The films were polycrystalline with columnar structure and a thickness around $340 \mathrm{~nm}$. From the diffraction patterns it can be deduced that the grains in the annealed films are bigger. An interfacial amorphous layer is present on both images with an average thickness of $1.4 \mathrm{~nm}$. This corresponds to the native silicon oxide, which was not removed prior to the deposition of the yttrium oxide layer. However, it is interesting to notice that the thickness of this layer does not alter significantly after annealing in vacuum. Recently Dimoulas et al. ${ }^{17}$ reported on the effects of the annealing process in vacuum of ultrathin $\mathrm{Y}_{2} \mathrm{O}_{3}$ films grown epitaxially on $\mathrm{Si}$. In some cases it was even possible to eliminate this native oxide film after annealing. Our re-

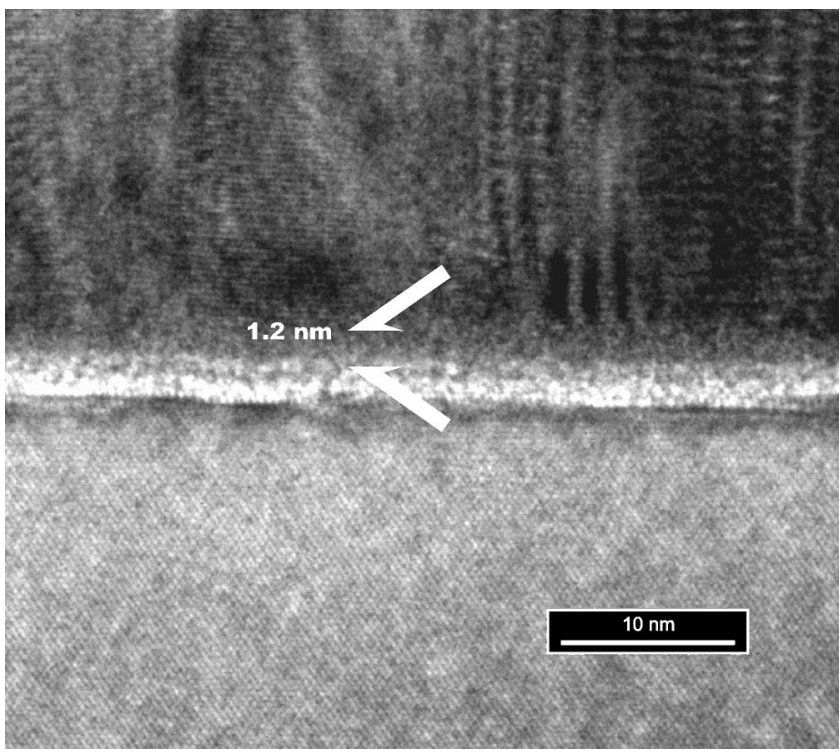

FIG. 3. HRTEM picture of the film annealed at $600{ }^{\circ} \mathrm{C}$. The $\mathrm{Y}_{2} \mathrm{O}_{3} / \mathrm{SiO}_{2}$ interface seems smoother with respect to the as deposited. The thickness of the native oxide is estimated around $1.2 \mathrm{~nm}$. 


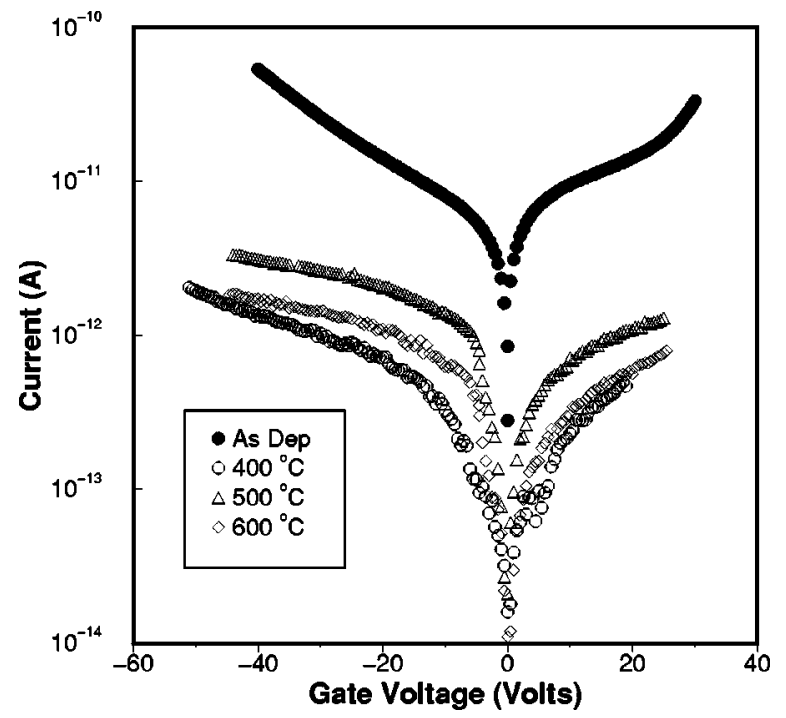

FIG. 4. $\log (I)-V$ characteristics of $\mathrm{Al} / \mathrm{Y}_{2} \mathrm{O}_{3} / \mathrm{Si}$ diodes. Films annealed at $400{ }^{\circ} \mathrm{C}$ showed the smallest leakage currents. A linear plot shows the ohmic behavior of the $I-V$ curves.

sults support their findings in the sense that although the as-deposited films are polycrystalline with a lot of grain boundaries and very thick, after annealing for $60 \mathrm{~min}$ in vacuum there is no formation of a thicker silicon oxide interfacial layer. We suppose that it was very difficult to eliminate it due to the thickness of the yttrium oxide layer. But if the diffusion of oxygen through the dielectric is enhanced due to the presence of the grain boundaries one might expect the amorphous silicon oxide interfacial layer to become thicker. A number of works can be found in the literature supporting this oxygen diffusion. ${ }^{21,23}$ Such an increase of the interfacial layer of the lower dielectric constant is highly unfavorable for the modern CMOS technology. Therefore our results are interesting as they indicate that the formation of this layer can be avoided after annealing in vacuum. In addition, it allows us to investigate the electrical characteristics of the $\mathrm{Si} / \mathrm{Y}_{2} \mathrm{O}_{3}$ interfaces without the additional problems introduced by a lower- $k \mathrm{SiO}_{x}$ layer grown in an uncontrolled way.

\section{Electrical characterization}

\section{Current density measurements}

The effect of crystallization and subsequent annealing of the as-deposited insulating $\mathrm{Y}_{2} \mathrm{O}_{3}$ films on the current leakage through the MIS structure is depicted in Fig. 4. The $I-V$ curves are almost identical irrespectively of the polarity of the gate voltage. This implies that the conduction mechanism is bulk limited. An interesting result is that the leakage current of the as-deposited film is much higher than that of the annealed films. In addition a dependence of the leakage current with the annealing temperature is evident, with the film annealed at $400{ }^{\circ} \mathrm{C}$ giving the best results. This behavior has also been reported on $\mathrm{Y}_{2} \mathrm{O}_{3}$ films of various thicknesses grown by a variety of techniques. ${ }^{6,8,9,17,23}$ Generally it is attributed to the different crystallinity of the films. It was usually accepted that due to the increased roughness of the sur- face as well as of the interface, greater leakage currents are expected. The reason for this is the appearance of weak spots at the contacts, which act as high-field regions and induce increased current conduction. However, a number of recent publications on alternative high- $k$ dielectrics ${ }^{3,10,24}$ show that even for very thin films this is not always true and some polycrystalline films show considerably lower leakage currents than the amorphous or epitaxial films. One possible explanation of the effect is related to the different conduction mechanisms involved. For the annealed films, it was found that the main conduction mechanism is that of electronic hopping conduction as can be deduced from the linear form of the $I-V$ curves (not shown in Fig. 4 for clarity reasons). One possible reason is that the local field is not high enough for the activation of the Poole-Frenkel mechanism (as, for example, in Ref. 8).

It should be mentioned here that the corresponding fields of the voltage values shown in Fig. 4 are up to $1 \mathrm{MV} \mathrm{cm}^{-1}$. No breakdown has been observed up to field values as high as $2 \mathrm{MV} \mathrm{cm}^{-1}$ as it was expected for the thick films we studied.

\section{Capacitance-voltage measurements}

The high frequency $(1 \mathrm{MHz})$ capacitance vs voltage $(C-V)$ characteristics are depicted in Fig. 5(a). In all cases the behavior of a typical MIS device was observed for voltage sweeps from inversion to accumulation, while sweep rates of $200 \mathrm{mV} \mathrm{s}^{-1}$ approximately have been used. The asdeposited films show a pronounced stretchout effect on the $C-V$ curves, which is also observed on the films annealed at $400{ }^{\circ} \mathrm{C}$, while the corresponding conductance-voltage $(G-V)$ curves [Fig. 5(b)] are very broad. The dielectric constant is also higher (15) for the as-deposited samples while for the annealed samples this value reduces to 14 . The dispersion of the capacitance values at inversion is probably due to defects at the interfaces, as from the TEM measurements we do not find any notable change of the thickness of the oxide. Therefore we could obtain accurate values for the doping concentration of the $\mathrm{Si}$ substrates (around 3 $\times 10^{15} \mathrm{~cm}^{-3}$ ) only for the films annealed at 500 and $600{ }^{\circ} \mathrm{C}$.

We would like to point out here that no quasistatic curves could be obtained mainly due to the high density of interface states as we explain in the next paragraphs. Therefore our analysis was based on the high frequency $C-V$ characteristics.

The flatband voltage $\left(V_{\mathrm{FB}}\right)$ in a $C-V$ curve is generally determined by the metal semiconductor work function difference $\phi_{\mathrm{ms}}$ and the various oxide charges through the relation:

$$
V_{\mathrm{FB}}=\phi_{\mathrm{ms}}-\frac{Q_{f}}{C_{\mathrm{ox}}}-\frac{Q_{\mathrm{it}}\left(\phi_{s}\right)}{C_{\mathrm{ox}}},
$$

where $Q_{f}$ is used to account for the fixed charge located very near the $\mathrm{Si} / \mathrm{SiO}_{2}$ interface as well as for the mobile and oxide charges. $^{25,26}$ Moreover, charges due to the interface traps $\left(Q_{\mathrm{it}}\right)$ can also contribute to a shift of the corresponding flatband voltage. It is therefore usual to acquire the $C-V$ curve of a MOS diode at frequencies sufficiently high so that the interface traps do not respond. Thus the last term in Eq. (1) is 

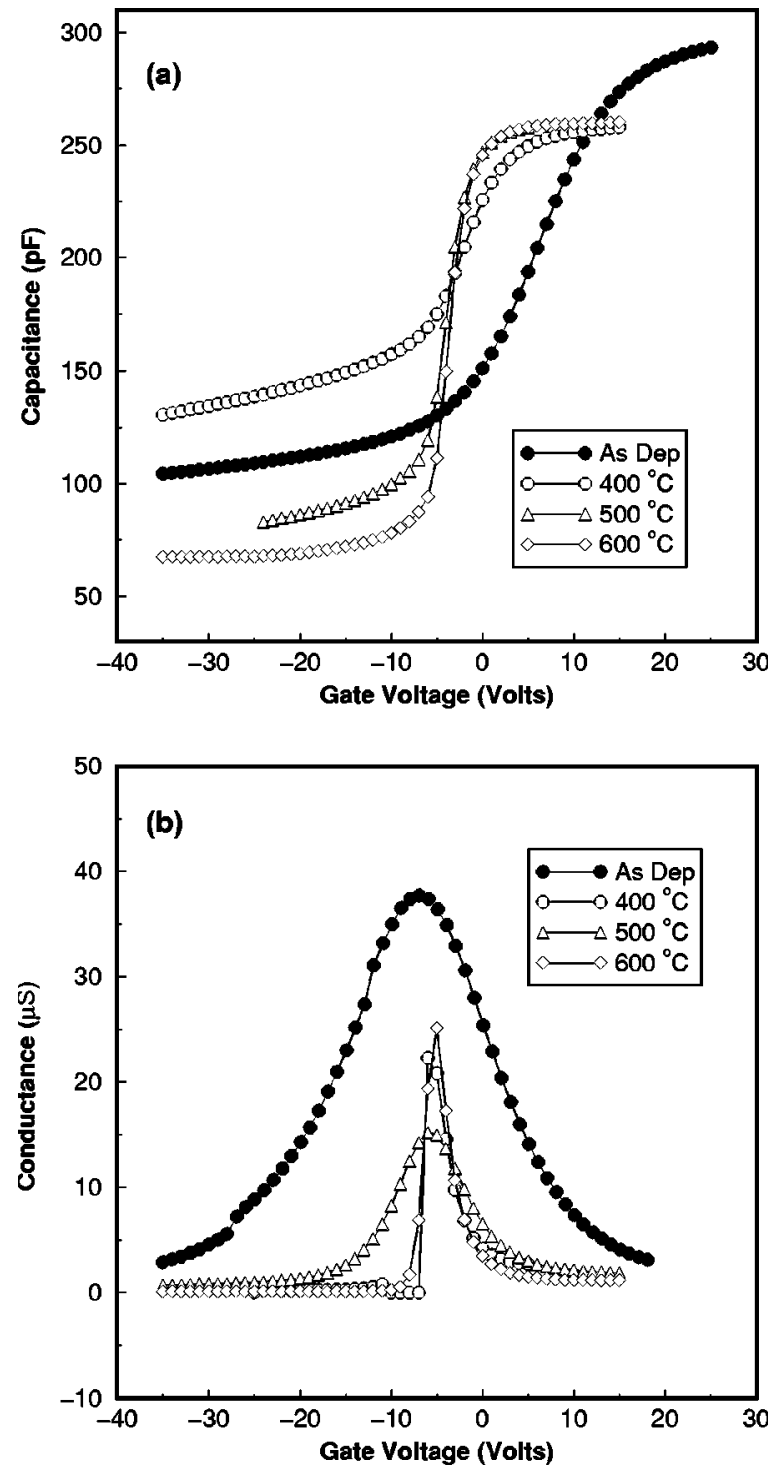

FIG. 5. High frequency (a) $C-V$ and (b) $G-V$ curves of the MOS capacitors. The flatband voltage shifts towards negative values after annealing.

omitted and the amount of fixed charge can be obtained. The $1 \mathrm{MHz}$ frequency used in our experiments was not sufficient in the case of the as-deposited film and the film annealed at $400{ }^{\circ} \mathrm{C}$. The relevant $C-V$ curves are "stretched out" and this can only be attributed to the high concentration of interface traps. Therefore the amount of trapped charge within the oxide layer was calculated only for the films annealed at 500 and $600{ }^{\circ} \mathrm{C}$ (Table I), where a negligible contribution from the interface traps is assumed.

TABLE I. Electrical parameters of the $\mathrm{Al} / \mathrm{Y}_{2} \mathrm{O}_{3} / \mathrm{Si}$ diodes.

\begin{tabular}{ccccc}
\hline \hline $\begin{array}{c}\mathrm{Ta} \\
\left({ }^{\circ} \mathrm{C}\right)\end{array}$ & $\varepsilon$ & $\begin{array}{c}D_{\mathrm{it}} \\
\left(\times 10^{12} \mathrm{eV}^{-1} \mathrm{~cm}^{-2}\right)\end{array}$ & $\begin{array}{c}V_{\mathrm{Fb}} \\
(\mathrm{V})\end{array}$ & $\begin{array}{c}Q_{f} \\
\left(\times 10^{11} \mathrm{~cm}^{-2}\right)\end{array}$ \\
\hline & 14.5 & 3.7 & +11 & \\
400 & 13.5 & 1.8 & -1 & \\
500 & 13.5 & 0.65 & -3 & 0.2 \\
600 & 13.5 & 0.70 & -2.5 & 3 \\
\hline \hline
\end{tabular}

Considerable displacement of the $V_{\mathrm{FB}}$ values is observed in the dielectrics before and after annealing. The origin of the positive charges in the $\mathrm{Y}_{2} \mathrm{O}_{3}$ dielectric is attributed to the inclusion of a large density of defects primarily from oxygen vacancies and broken bonds. It seems though that an annealing temperature of around $500{ }^{\circ} \mathrm{C}$ represents a threshold of oxygen intake in the $\mathrm{Y}_{2} \mathrm{O}_{3}$ film, which reduces considerably the oxygen-related defects and restores compositional homogeneity of the $\mathrm{Y}_{2} \mathrm{O}_{3}$ dielectric, resulting in reduction of the positive charge density. This result seems to be independent of the growth technique used as it has been also reported in the past for electron-beam deposited films. ${ }^{8-10}$

The corresponding conductance vs voltage $(G-V)$ curves at $100 \mathrm{KHz}$ are shown in Fig. 5(b). The interface state densities $D_{\text {it }}$ were calculated from the $C-V$ and $G-V$ plots [Figs. 5(a) and 5(b)] using the following relation of Hill's method: $:^{27}$

$$
D_{\mathrm{it}}=\frac{2}{q A}\left(\frac{G_{\mathrm{max}}}{\omega}\right)\left[\left(\frac{G_{\mathrm{max}}}{\omega C_{\mathrm{ox}}}\right)^{2}+\left(1-\frac{C_{\mathrm{max}}}{C_{\mathrm{ox}}}\right)^{2}\right]^{-1},
$$

where $D_{\text {it }}$ is the interface state density, $A$ is the area of the capacitor, $q$ the electronic charge, and $C_{\mathrm{ox}}$ is the capacitance in accumulation. $G_{\max }$ is the peak value of the conductance (obtained from the $G-V$ curves) and $C_{\max }$ is the capacitance corresponding to $G_{\max }$. The $C-V$ and $G-V$ plots at 100 $\mathrm{KHz}$ have been chosen so that no correction was required on both $C$ and $G$ measured values [Fig. 5(b)]. Thus the minimum $D_{\text {it }}$ values calculated were in the order of $10^{12} \mathrm{eV}^{-1} \mathrm{~cm}^{-2}$. Recently, the Terman's ${ }^{25,26}$ method gained some attention for the study of high- $k$ oxides, as in most cases the observed density of interface states is greater than $10^{11} \mathrm{eV}^{-1} \mathrm{~cm}^{-2}$. From the analysis of the high frequency $C-V$ curves according to this method we obtained consistently higher $D_{\text {it }}$ values than the Hill's method for all samples, but always in the range of $(2-8)$ $\times 10^{12} \mathrm{eV}^{-1} \mathrm{~cm}^{-2}$. This high density of interface states is probably the main reason that we were not able to obtain quasistatic $C-V$ plots. Therefore, at this stage, we have an indication of high $D_{\mathrm{it}}$ values for the studied structures although for real applications these values have to be evaluated after proper annealing in forming gas.

\section{Hysteresis effects}

A significant effect of annealing is the change of the direction of the hysteresis loop. Hysteresis is measured by first driving the MIS diode from strong inversion to accumulation and backwards. The rate of the voltage ramp was always around $200 \mathrm{mV} \mathrm{s}^{-1}$. Normalized $C / C_{\text {ox }}$ versus gate voltage curves of the diodes containing the as-deposited and the film annealed at $600^{\circ} \mathrm{C}$ are illustrated in Fig. 6. It is interesting to point out that only the as-deposited films as well as the films annealed at $400{ }^{\circ} \mathrm{C}$ show a notable hysteresis curve $\left(\Delta V_{H}>3 \mathrm{~V}\right.$ in the case of the as-deposited films). The film annealed at $400{ }^{\circ} \mathrm{C}$ is the only one showing clockwise direction of the hysteresis curve which could be attributed to electron injection in accumulation. On the other hand, the relevant shifts $\Delta V_{H}$ are $<0.20$ and $<0.10 \mathrm{~V}$ for the films annealed at 500 and $600{ }^{\circ} \mathrm{C}$, respectively. 


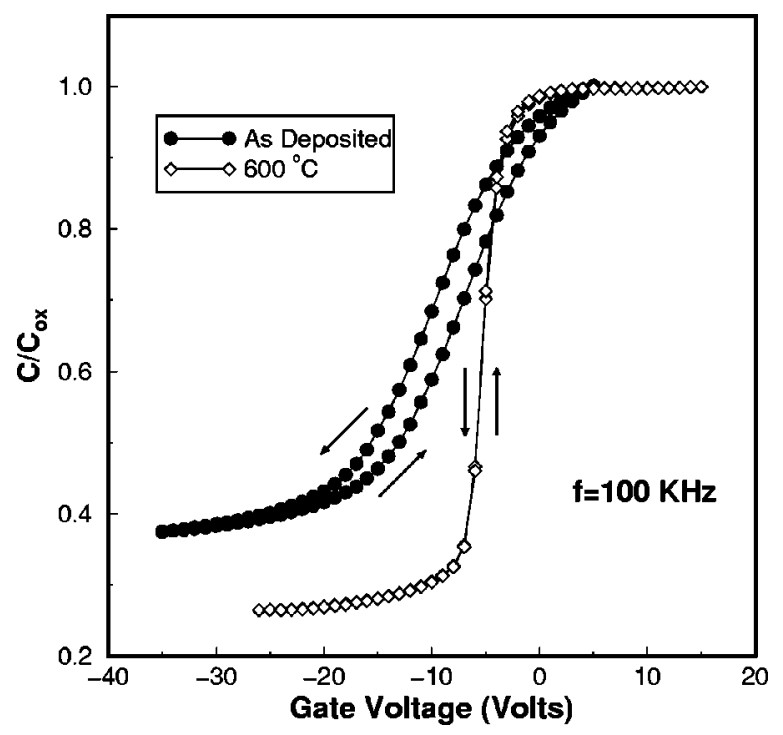

FIG. 6. $C-V$ curves showing hysteresis effects. Hysteresis of the films annealed at $600{ }^{\circ} \mathrm{C}$ is small $(<100 \mathrm{mV})$ as compared to the as-deposited films $(>3 \mathrm{~V})$.

From the preceding analysis and the $D_{\text {it }}$ and $Q_{f}$ values reported in Table I, the hysteresis results can be directly related to the amount of defects present in the oxide as well as at the $\mathrm{Y}_{2} \mathrm{O}_{3} / \mathrm{SiO}_{2}$ interface. The origin of this effect is still unclear. Nevertheless, it is anticipated that the observed counterclockwise loops may be due to a polarization in the $\mathrm{Y}_{2} \mathrm{O}_{3} / \mathrm{SiO}_{2}$ interface, which introduces a sheet of positive charge. The magnitude of this charge is expected to be dependent on the field strength in the film. ${ }^{28}$ Indeed, a change of the voltage ramp or the maximum voltage in accumulation results in observable changes in the relevant $\Delta V_{H}$ values. In addition, the higher annealing temperature leads to a better, from the electrical point of view, $\mathrm{Y}_{2} \mathrm{O}_{3} / \mathrm{SiO}_{2}$ interface so the effect almost disappears. On the other hand, the high density of defects present within the oxide may also give rise to this enhanced hysteresis effect. It has been reported that this hysteresis is related to oxygen deficiency and is also thickness dependent. ${ }^{29}$ Therefore in addition to the above-mentioned $\mathrm{Y}_{2} \mathrm{O}_{3} / \mathrm{SiO}_{2}$ interface charge we assume that it is also the reduction in bulk oxygen-related defects that contributes towards the minimization of hysteresis with the annealing temperature.

An important implication of the inherent formation of electron traps at the high- $k$ oxide/ $/ \mathrm{SiO}_{2}$ interface is that they capture the injected electrons and as a result a reduced leakage current in MIS diodes with bilayer dielectric has been reported. ${ }^{18,30}$ Indeed the large reduction in the leakage current conduction through the $c-\mathrm{Y}_{2} \mathrm{O}_{3} / \mathrm{Si}$ structures observed after annealing at $400{ }^{\circ} \mathrm{C}$ (Fig. 4) is understood on this basis.

Finally, we would like to stress that a very interesting outcome from the preceding analysis is that the defects present in our films are located close to the silicon interface within the bulk of the oxide and reduce considerably after annealing at $600{ }^{\circ} \mathrm{C}$ in vacuum. This will be used as a starting point for the analysis of the $C-V$ shifts with frequency in the next section.

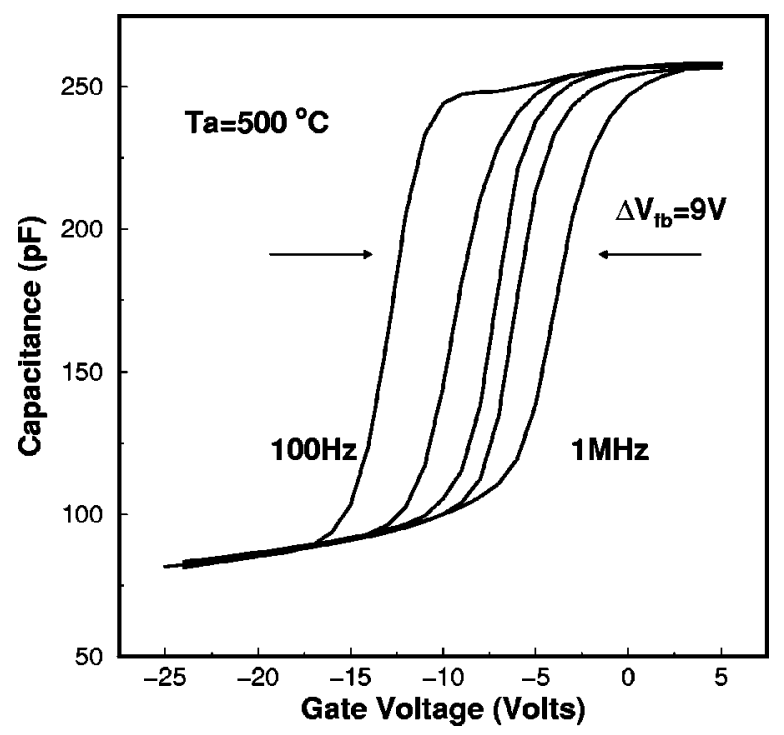

FIG. 7. Shift of the $C-V$ curves with frequency for the films annealed at $500^{\circ} \mathrm{C}$. The flatband voltage shift $\Delta V_{\mathrm{FB}}$ marked in the figure is greater than $30 \mathrm{~V}$ for the as-deposited films and reduces considerably with the annealing temperature.

\section{Frequency dependence of the $C-V$ curves}

An interesting feature of the diodes is that the flatband voltage shifts towards more positive values with increasing frequency of the applied ac signal. In Fig. 7 this effect is illustrated for the films annealed at $500^{\circ} \mathrm{C}$. The transition from inversion to accumulation is sharp and the capacitance in both inversion and accumulation regimes $\left(C_{\text {acc }}\right.$ and $\left.C_{\text {inv }}\right)$ is almost identical for all measured frequencies. The effect is clearly observed in all films but is more profound for the as-deposited films leading to a flatband voltage shift $\Delta V_{\mathrm{FB}}$ $>35 \mathrm{~V}$ where we define $\Delta V_{\mathrm{FB}}$ as

$$
\Delta V_{\mathrm{FB}}=V_{\mathrm{FB}}(100 \mathrm{~Hz})-V_{\mathrm{FB}}(1 \mathrm{MHz}) .
$$

These characteristics are difficult to explain on the basis of the interface model developed for the $\mathrm{Si} / \mathrm{SiO}_{2}$ interface. ${ }^{25,26}$ If a large density of interface states is present at the interface, then the $C-V$ curves are "stretched out" with frequency. The capacitance in inversion is not affected but a small dispersion in $C_{\text {acc }}$ may arise. In some extreme cases $\left(D_{\mathrm{it}}>10^{13} \mathrm{eV}^{-1} \mathrm{~cm}^{-2}\right)$ a greater dispersion in $C_{\text {acc }}$ values has been reported ${ }^{31}$ together with a clear shift of $V_{\mathrm{FB}}$ with increasing frequency. However, the use of $\mathrm{Si}$ (111) substrate is probably the main cause for this elevated density of interface states in this rather old work. During the last two decades the quality of $\mathrm{SiO}_{2}$ films and the corresponding $\mathrm{Si} / \mathrm{SiO}_{2}$ interface have improved considerably. The presence of any interface states lead to $C-V$ curves which are "stretched out" at lower frequencies and become abrupt at high $(0.1-1 \mathrm{MHz})$ frequencies. Therefore a number of techniques have been developed ${ }^{25,26}$ to translate this change in slope of the $C-V$ curves in the depletion region to a graph of $D_{\text {it }}$ as a function of the applied gate voltage $V_{g}$ (or surface potential $\phi_{s}$ or energy in Si band gap). An additional parallel shift of the $C-V$ curves occurs when a fixed charge is trapped $\left(Q_{f}\right)$ in the oxide. The amount of fixed charge can be 
related to the measured $V_{\mathrm{FB}}$ by Eq. (1). In the present work though, the $C-V$ plot is shifted along the voltage axis with frequency but not stretched.

To the best of our knowledge only a few works have been reported in the past ${ }^{14,31-34}$ showing a parallel shift of measured $C-V$ 's with frequency. An interesting common feature in these works is that all of them report on interfaces between silicon and poor dielectric materials [e.g., $\mathrm{ZnS}: \mathrm{Mn}$ (Ref. 32) or amorphous carbon (Ref. 33)]. In the case of Refs. 32 and 34, the authors do not make an attempt to explain the effect. On the other hand Khan et al. ${ }^{33}$ do explain their results using a model based on carrier tunneling into insulator states. ${ }^{35}$ In their case though, $V_{\mathrm{FB}}$ shifts towards more negative voltages with increasing frequency and a very low (around $60 \mathrm{~Hz}$ ) frequency is needed in order to obtain near ideal high frequency $C-V$ curves. Nevertheless, their results can be directly related to ours in the sense that the corresponding $V_{\mathrm{FB}}$ shifts towards accumulation with increasing frequency.

We therefore propose a model to elucidate our results based on the same earlier theoretical work by Heiman and Warfield. ${ }^{35}$ The concept of this model is that a number of defects (states) introduced uniformly into the bulk of the oxide and close to the interface with silicon have an exponential distribution of time constants with depth in the insulator. At a given frequency all traps with time constants shorter than the reciprocal of the frequency are able to follow the ac signal, thus the flatband condition is reached at a certain gate voltage. If now the frequency of the measurement is increased, slower traps will not be able to respond to the measuring signal and will stay (negatively) charged; hence flatband conditions will be met at more positive gate voltages. Work is currently under progress to fit this model to the experimental conductance vs frequency $(G p / \omega-\omega)$ data.

Therefore according to this model we can conclude from the present work that the amount of oxide traps reduces considerably with the annealing temperatures as implied from the reduction of $\Delta V_{\mathrm{FB}}$ with the annealing temperature. Indeed, $\Delta V_{\mathrm{FB}}$ values greater than $30 \mathrm{~V}$ were observed on the as-deposited films but reduce to $6 \mathrm{~V}$ for the films annealed at $600{ }^{\circ} \mathrm{C}$.

In an attempt to comment on the origin of these defects we believe that they are related to the oxygen vacancies in the oxide and/or states at the interface between native oxide and $\mathrm{Y}_{2} \mathrm{O}_{3}$. We believe that the existence of discrete level oxygen vacancies with uniform spatial distribution fits better to the proposed model but a definite answer will be given only after studying an oxide/Si interface free from the native oxide interfacial layer.

\section{CONCLUSIONS}

The main aim of this work was to study the interface properties of rf-magnetron sputtered yttrium oxide films on $n$-Si(100) subjected to postdeposition annealing at temperatures in the range $400-600{ }^{\circ} \mathrm{C}$ in vacuum. For this purpose rather thick films (around $340 \mathrm{~nm}$ ) have been deposited in order to obtain extremely low leakage currents.
$\mathrm{X}$-ray diffraction analysis revealed a mixture of yttrium suboxides and cubic $\mathrm{Y}_{2} \mathrm{O}_{3}$ in the as-grown film. Upon annealing the $\mathrm{Y}_{2} \mathrm{O}_{3}$ cubic phase crystallites are bigger and strongly oriented with the (111) planes parallel to substrate. A thin native oxide layer is present both in the as-deposited and the annealed films. HRTEM pictures show clearly that this interfacial layer remains almost constant even after annealing at $600{ }^{\circ} \mathrm{C}$.

The dielectric constant of the annealed films was around 13.5 and did not change with the annealing temperature. The electrical characterization of the corresponding MOS devices revealed a mixture of interface and bulk oxygen related defects that give rise to an enhanced shift of the $C-V$ curves along the voltage axis with frequency. An attempt to explain this effect was given based on the response of these defects to the ac signal. The $D_{\text {it }}$ values are obviously too high for the devices to be considered as potential alternatives to the current silicon oxide based CMOS technology. A different annealing procedure is needed if this material is to be used as an alternative gate dielectric. On the other hand, if the effects of the bulk oxide defects are thickness dependent they are not expected to cause significant problems on more realistic CMOS applications $\left(t_{\mathrm{ox}} \sim 5 \mathrm{~nm}\right)$. In any case, the study of these defects in films of variable thickness is needed in order to understand their origin and suggest possible ways of elimination.

${ }^{1}$ M. L. Green, E. P. Gusev, R. Degraeve, and E. L. Garfunkel, J. Appl. Phys. 90, 2057 (2001)

${ }^{2}$ H. Iwai and S. Ohmi, Microelectron. Reliab. 42, 465 (2002).

${ }^{3}$ G. D. Wilk, R. M. Wallace, and J. M. Anthony, J. Appl. Phys. 89, 5243 (2001); D. A. Buchanan, IBM J. Res. Dev. 43, 245 (1999).

${ }^{4}$ E. Evangelou, N. Konofaos, and C. B. Thomas, Philos. Mag. B 80, 395 (2000); Z. Wang, V. Kugler, U. Helmersson, N. Konofaos, and E. K. Evangelou, ibid. 82, 891 (2002).

${ }^{5}$ W. M. Cranton, D. M. Spink, R. Stevens, and C. B. Thomas, Thin Solid Films 226, 156 (1993).

${ }^{6}$ R. H. Horng, D. S. Wuu, J. W. Yu, and C. Y. Kung, Thin Solid Films 289, 234 (1996).

${ }^{7}$ A. F. Jankowski, L. R. Schrawyer, and J. P. Hayer, J. Vac. Sci. Technol. A 11, 1548 (1993)

${ }^{8}$ A. C. Rastogi and R. N. Sharma, J. Appl. Phys. 71, 5041 (1992).

${ }^{9}$ A. C. Rastogi and R. N. Sharma, Semicond. Sci. Technol. 16, 641 (2001).

${ }^{10}$ A. Dimoulas, A. Travlos, G. Vellianitis, N. Boukos, and K. Argyropoulos, J. Appl. Phys. 90, 4224 (2001).

${ }^{11}$ S. Zang and R. Xiao, J. Appl. Phys. 83, 3842 (1998).

${ }^{12}$ H. J. Gao, D. Kummar, K. G. Cho, P. H. Holloway, R. K. Singh, X. D. Fan, Y. Yan, and S. J. Pennycook, Appl. Phys. Lett. 75, 2223 (1999).

${ }^{13}$ M. Putkonen, T. Sajavaara, L. S. Johansson, and L. Niinisto, Chem. Vap. Deposition 7, 44 (2001).

${ }^{14}$ L.-A. Ragnarsson, S. Guha, M. Copel, E. Cartier, N. A. Bojarczuk, and J. Karasinski, Appl. Phys. Lett. 78, 4169 (2001).

${ }^{15}$ K. J. Hubbard and D. G. Schlom, J. Mater. Res. 11, 2757 (1996).

${ }^{16}$ J. Robertson, MRS Bull. 27, 217 (2002).

${ }^{17}$ A. Dimoulas, G. Vellianitis, A. Travlos, V. Ioannou, and A. G. Nasiopoulou, J. Appl. Phys. 92, 426 (2002).

${ }^{18}$ R. S. Johnson, G. Lukovsky, and J. G. Hong, Microelectron. Eng. 59, 385 (2001).

${ }^{19}$ H. Fukumoto, T. Imura, and Y. Osaka, Appl. Phys. Lett. 55, 360 (1989).

${ }^{20}$ K. Harada, H. Nakanishi, H. Itozaki, and S. Yazu, Jpn. J. Appl. Phys., Part 1 30, 934 (1991).

${ }^{21}$ S. C. Choi, M. H. Cho, S. W. Whangbo, C. N. Whang, S. B. Kang, S. I. Lee, and M. Y. Lee, Appl. Phys. Lett. 71, 903 (1997).

${ }^{22}$ R. J. Gaboriaud, F. Pailloux, P. Guerin, and F. Paumier, J. Phys. D 33, 2884 (2000)

${ }^{23}$ J. Kwo, M. Hong, A. R. Kortan, K. L. Queeney, Y. J. Chabal, R. L. Opila, Jr., D. A. Muller, S. N. G. Chu, B. J. Sapjeta, T. S. Lay, J. P. Mannaerts, T. 
Boone, H. W. Krautter, J. J. Krajewski, A. M. Sergnt, and J. M. Rosamilia, J. Appl. Phys. 89, 3920 (2001).

${ }^{24}$ S. Guha, E. Cartier, M. A. Gribelyuk, N. A. Bojarczuk, and M. Copel, Appl. Phys. Lett. 77, 2710 (2000).

${ }^{25}$ D. Schroder, Semiconductor Material and Device Characterization, 2nd ed. (Wiley, New York, 1998).

${ }^{26}$ E. H. Nicollian and J. R. Brews, MOS Physics and Technology (Wiley, New York, 1982).

${ }^{27}$ W. A. Hill and C. C. Coleman, Solid-State Electron. 23, 987 (1980).

${ }^{28}$ C. H. Ling, J. Bhaskaran, W. K. Choi, and L. K. Ah, J. Appl. Phys. 77, 6350 (1995).

${ }^{29}$ S. Ramanathan, C.-M. Park, and P. McIntyre, J. Appl. Phys. 91, 4521
(2002), and references therein.

${ }^{30}$ J. P. Maria, D. Wicaksana, A. I. Kingon, B. Bush, H. Schulte, E. Garfunkel, and T. Gustafsson, J. Appl. Phys. 90, 3476 (2001).

${ }^{31}$ J. P. Courat, G. Nugillat, and P. Sixou, Solid State Commun. 7, 1123 (1969).

${ }^{32}$ A. J. Simons, M. H. Tayarani-Najaran, and C. B. Thomas, J. Appl. Phys. 70, 1 (1991).

${ }^{33}$ A. A. Khan, J. A. Woollam, and Y. Chung, J. Appl. Phys. 55, 4299 (1984).

${ }^{34}$ H. Harris, N. Biswas, H. Temkin, S. Gangopadhyay, and M. Strathman, J. Appl. Phys. 90, 5825 (2001).

${ }^{35}$ F. Heiman and G. Warfield, IEEE Trans. Electron Devices ED-12, 167 (1965). 